\title{
Robust Spectrum Sensing Demonstration Using a Low-Cost Front-End Receiver
}

\author{
Daniele Borio, Emanuele Angiuli, Raimondo Giuliani, and Gianmarco Baldini
}

European Commission, Joint Research Centre (JRC) Institute for the Protection and Security of the Citizen (IPSC)

Digital Citizen Security Unit, Via Enrico Fermi 2749, 21027 Ispra, Italy

Correspondence should be addressed to Gianmarco Baldini; gianmarco.baldini@jrc.ec.europa.eu

Received 19 April 2015; Revised 3 July 2015; Accepted 9 July 2015

Academic Editor: Feifei Gao

Copyright (C) 2015 Daniele Borio et al. This is an open access article distributed under the Creative Commons Attribution License, which permits unrestricted use, distribution, and reproduction in any medium, provided the original work is properly cited.

\begin{abstract}
Spectrum Sensing (SS) is an important function in Cognitive Radio (CR) to detect primary users. The design of SS algorithms is one of the most challenging tasks in CR and requires innovative hardware and software solutions to enhance detection probability and minimize low false alarm probability. Although several SS algorithms have been developed in the specialized literature, limited work has been done to practically demonstrate the feasibility of this function on platforms with significant computational and hardware constraints. In this paper, SS is demonstrated using a low cost TV tuner as agile front-end for sensing a large portion of the UltraHigh Frequency (UHF) spectrum. The problems encountered and the limitations imposed by the front-end are analysed along with the solutions adopted. Finally, the spectrum sensor developed is implemented on an Android device and SS implementation is demonstrated using a smartphone.
\end{abstract}

\section{Introduction}

Spectrum Sensing (SS) is one of the most challenging tasks in Cognitive Radio (CR) [1]. In particular, SS consists of scanning a large portion of the Radio Frequency (RF) spectrum and detecting the presence of possibly weak signals in the frequency band of interest. A cognitive device has to determine with a high level of confidence if a specific band is unoccupied before using it for its own operations. High detection probability is required to ensure that primary users are adequately protected, while minimization of false alarms improves the efficiency of secondary users to transmit in RF spectrum bands not occupied by primary users. For this reason, several techniques have been developed to improve the sensitivity of SS. These techniques include the energy detector, the cyclostationarity detector and the matched filter detector [1-3]. The energy detector is the most simple technique available in the literature because of its low computational and implementation complexity. In addition, the energy detector does not require a priori knowledge of the primary user Signal-ofInterest (SOI) and it is therefore more versatile and it can be adopted for the different types of signals. These techniques are designed assuming an ideal front-end for the collection of the samples used for signal detection. Thus, the presence of spurs and gain variations are usually neglected. The oscillator used for signal down-conversion can however introduce harmonics and power can "leak" from adjacent bands if the front-end filter has insufficient side-band rejection. Frontend nonidealities can strongly impact the detection process and need to be accounted for. Although these nonidealities are considered in the recent literature [4-6], only ad hoc solutions, including the increase of the decision threshold $[4,5]$ and the usage of frequency domain excision techniques [6], were suggested.

In this paper, practical SS is demonstrated using a lowcost TV tuner, the Realtek RTL2832U [7] device, which is adopted as agile front-end. The nonlinearities caused by the low-cost device are accounted for using a robust approach for spurious removal. The technique proposed requires a reduced computation load and can thus be implemented on devices with limited computational resources.

The contribution of this paper is twofold. At first the propagation impairments caused by a low-cost front-end are experimentally characterized. It is shown that SS has to take 
into account the spurs and interfering signals caused by the front-end, that is, the sensor used for sensing. Traditional techniques such as the energy detector can be negatively affected by the presence of such interfering terms and robust detection techniques [8] should be employed. In this respect, the classical energy detector is modified using robust operators such as the median. The development of robust energy detection techniques is the second major contribution of the paper. The advantages of the technique developed are analysed in terms of probability of false alarm, that is, the probability of incorrectly declaring a frequency band occupied. The use of the median as robust operator allows the removal of spurious components improving the overall performance of the system.

The technique proposed is computationally efficient and can be implemented in platforms with limited computational resources. In this respect, the developed SS technique has been implemented on an Android device and SS is demonstrated using a smartphone.

The remainder of the paper is organized as follows: the problem of SS and classical SS techniques are discussed in Section 2 whereas the signal model adopted for the detector design is provided in Section 3. The robust energy detector proposed to deal with interference is described in Section 4 and characterized from a statistical point of view in Section 5. The system architecture and its implementation are detailed in Section 6. Experimental results are finally provided in Section 7. Section 8 concludes the paper.

\section{Spectrum Sensing Techniques}

SS is the process of detecting the signal presence in a specific frequency band. Thus, it is a binary hypothesis testing problem where it is necessary to decide between

$$
\begin{aligned}
& H_{0}: y[n]=\eta[n], \quad n=0, \ldots, N-1, \\
& H_{1}: y[n]=x[n]+\eta[n], \quad n=0, \ldots, N-1,
\end{aligned}
$$

where $H_{0}$ and $H_{1}$ are the null and alternative hypothesis, respectively. The null hypothesis assumes that the band of interest (portion of spectrum under analysis) is not occupied by a signal component and only the noise term, $\eta[n]$, is present. On the contrary, the alternative hypothesis, $H_{1}$, assumes that also a useful signal component, $x[n]$, is present. In (1), the different components are digital sequences obtained by digitizing an analog signal using a sampling frequency, $f_{s}$.

Note that (1) defines the simplest form of the problem addressed by SS. In particular, under the assumption that $\eta[n]$ is a complex circularly symmetric Gaussian process, model (1) defines a simple Additive White Gaussian Noise (AWGN) channel. Effects such as interference and channel distortions are neglected. More complex models accounting for fading and interference are to be considered in order to handle real channel effects $[3,9]$. In this paper, a signal model accounting for frequency-dependent interference terms is considered. Moreover, flat spectral characteristics are assumed on the frequency band considered for sensing. More details on the model adopted in this paper are provided in Section 3.

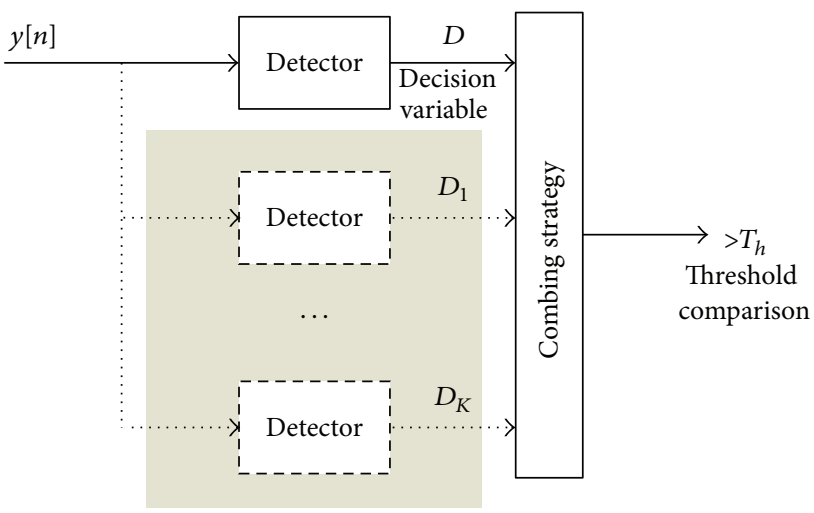

Figure 1: Principles of signal detection: the decision variable, $D$, is computed from the input sample, $y[n]$. Several detectors can be adopted in parallel to compute a set of decision variables. A combining strategy is then adopted to evaluate the final decision statistic.

In order to decide between $H_{0}$ and $H_{1}$, different detectors have been suggested in the literature [10,11]. A detector is a function of the input data which is used to compute a decision variable which, in turn, is compared with a decision threshold [12]. The basic principle of signal detection is shown in Figure 1. The input samples, $y[n]$, are combined to form the decision statistic, $D$. The alternative hypothesis is selected if $D$ passes the decision threshold, $T_{h}$. Note that several detectors can be adopted in parallel as shown in Figure 1. In this case, a combining strategy is required to form a new composite decision variable which is then compared against the decision threshold. The use of several detectors can improve the reliability of the final decision.

A review of the different detectors adopted in the literature for SS can be found in $[10,11]$. At the basis of the design of a signal detector, there is usually a model which incorporates the information available on the signal and noise components in (1). In general, the more information on the signal structure is exploited, the more the detector will be effective. This principle is valid only in the absence of model mismatches: if errors are present in the signal and noise descriptions then the detector may suffer significant performance degradations. Thus, the design of a signal detector is usually a compromise between detection performance and robustness against model mismatches.

A signal detector widely adopted in the literature is the energy detector:

$$
D_{e}=\frac{1}{N} \sum_{n=0}^{N-1}|y[n]|^{2},
$$

where $D_{e}$ represents an estimate of the energy of the input samples, $y[n]$. The assumptions behind (2) are that both signal and noise samples can be modelled as zero mean circularly symmetric Gaussian random processes.

When the energy detector is used, a signal in a specific frequency band is declared present if its energy exceeds a decision threshold, $T_{h}$. This threshold depends on the noise variance, $\sigma^{2}$, which should be known. When $\sigma^{2}$ is not known, 
it needs to be estimated. For this reason, noise floor estimators are required. A simple approach is to measure $\sigma^{2}$ by considering signals in frequency bands that are known to be unoccupied and contain noise only. Note that the problem of noise floor estimation is a common problem in communication and navigation. Examples of noise floor estimators adopted for the detection of Global Navigation Satellite System (GNSS) signals can be found in [13]. The basic principle of noise floor estimation is to remove first the signal component and measure the noise power alone. Errors in the knowledge of $\sigma^{2}$ can significantly reduce detection performance.

The energy detector has been used as starting point for the design of several SS techniques which account, for example, for communication channels with non-flat spectral characteristics [9] and for other transmission impairments [3].

More advanced detectors employed for SS exploit the knowledge of specific signal properties and are often referred to as feature detectors [10]. For example, communication signals are often characterized by nonstationary correlation functions (second order statistics) and detection can be performed in the signal correlation domain. These detectors include the cyclostationary detector and the peak autocorrelation magnitude detector. The former tries to identify the presence of periodic patterns in the time-varying autocorrelation function of the input sample, $y[n]$; the latter verifies if the maximum of the autocorrelation function (computed on a sliding window) is higher than the decision threshold. More recently, Compressive Sensing (CS) approaches have also been adopted for SS [14]. These techniques allow a significant reduction in the number of samples required for detecting the signal presence.

Several other detectors have been suggested and additional details can be found in $[10,11]$ and in the references therein.

\section{Signal Model}

Low-Cost Software Defined Radio (SDR) front-ends, such as the Realtek RTL2832U [7] device, are able to collect relatively narrowband signals with a specified centre frequency, $f_{c}$. In particular, the centre frequency of the band of interest can be easily specified using the Application Program Interface (API) of the device [7].

The signals are filtered, down-converted, sampled, and digitized. The output of the front-end is thus a digital sequence which can be effectively modelled as

$$
\begin{aligned}
y\left(n T_{s}, f_{c}\right)= & \sigma_{s} s\left(n T_{s}, f_{c}\right)+A\left(f_{c}\right) i\left(n T_{s}, f_{c}\right) \\
& +\eta\left(n T_{s}, f_{c}\right),
\end{aligned}
$$

where $T_{s}=1 / f_{s}$ is the sampling interval used to digitize the input analog signal. In (3), $s\left(n T_{s}, f_{c}\right)$ is the useful signal normalized in order to have real and imaginary parts with unit power. Depending on the SOI, different models can be adopted to describe $s\left(n T_{s}, f_{c}\right)$. Moreover, additional factors can be added in order to take into account propagation effects such as the channel impulse response. In this paper, narrowband SS is performed; that is, relatively small frequency bands are considered during sensing. In this way, it is possible to assume a flat frequency channel on the frequency band selected. This model is justified by the experimental results reported in Section 7.

When TV White Spaces (WSs) are considered, $s\left(n T_{s}, f_{c}\right)$ is modelled as a zero mean complex circularly symmetric Gaussian random process with real and imaginary parts with unit variance. This type of model is justified for Orthogonal Frequency Division Multiplexing (OFDM) modulations [15] where the central limit theorem can be applied. The symbol, $\sigma_{s}$, determines the total power, $C=2 \sigma_{s}^{2}$, of the useful signal. When $\sigma_{s}=0$, the useful signal components are absent and the channel is available for secondary users. Detection problem (1) can be restated in terms of $\sigma_{s}$ : the goal of SS is to determine if $\sigma_{s}$ is equal to zero or not.

Finally, it is noted that low-cost front-ends are characterized by relatively low sampling frequencies, $f_{s}$. Thus, $s\left(n T_{s}, f_{c}\right)$ represents only a narrow portion of the SOI. Components from several frequencies should be considered to detect the presence of wideband signals.

Signal $i\left(n T_{s}, f_{c}\right)$ is a complex interfering term with amplitude, $A\left(f_{c}\right)$, dependent on the centre frequency, $f_{c}$. It will be shown that, in the absence of interference from adjacent bands, $i\left(n T_{s}, f_{c}\right)$ can be effectively described as a Continuous Wave (CW). More complex models should be considered to account for the impact of receiver nonlinearities in the presence of strong signals on adjacent bands [4]. When $A=0$, no interference is present. $\eta\left(n T_{s}, f_{c}\right)$ is the receiver noise modelled as a zero mean complex circularly symmetric Gaussian random process with real and imaginary parts with variance, $\sigma^{2}\left(f_{c}\right)$.

The interference term is due to Local Oscillator (LO) leakage, In-Phase/Quadrature (I/Q) imbalances, and Direct Current (DC) offset. LO leakage occurs when the signal generated by the local oscillator enters the reception path of the front-end and thus a CW interference is perceived by the receiver. I/Q imbalances are due to a nonideal behaviour of the two arms of the front-end demodulator whereas the DC offset is due to power supply noise leaking on the demodulator output.

Standard SS techniques neglect the presence of the interfering term, $i\left(n T_{s}, f_{c}\right)$, and thus can suffer significant performance degradation. In the next section, an approach robust to frequency outliers is derived. An empirical justification to model (3) is provided in Section 7.

\section{Robust Energy Detection}

In order to be able to perform SS in the presence of interference, a robust algorithm is required. In this paper, robustness is intended in the distributional sense; that is, it is the ability of a system to deal with model mismatches [8]. Consider, for example, a simple energy detector, in the absence of signal, the input samples are assumed to be made of noise only. The noise term is assumed to follow a complex Gaussian distribution and the decision threshold is set according to this hypothesis. When oscillator spurs are present, this hypothesis is violated and the model is no longer valid. A robust SS technique has to be able to deal with this type of model mismatch. In addition to this, the algorithms have to be 


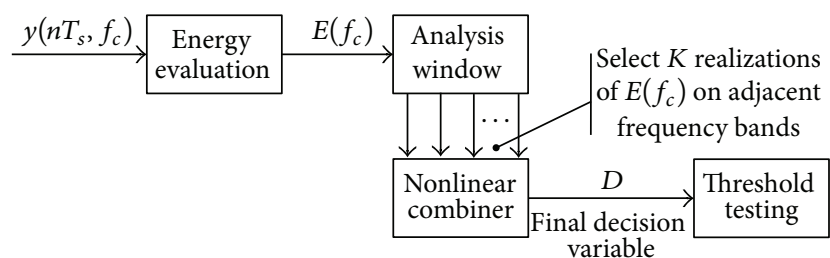

FIGURE 2: Two-stage detector architecture.

computationally efficient in order to be able to operate in realtime, for example, on an Android platform.

As mentioned in Section 2, the energy of a signal is defined as

$$
E\left(f_{c}\right)=\frac{1}{N} \sum_{n=0}^{N-1}\left|y\left(n T_{s}, f_{c}\right)\right|^{2} .
$$

This is the energy measured around the centre frequency, $f_{c}$, considering a narrow portion of the spectrum. This frequency band is dictated by the sampling capabilities of the front-end. If the SOI is wideband, then its total energy is given by

$$
D_{e}=\frac{1}{K} \sum_{k=0}^{K-1} E\left(f_{\min }+k \Delta f\right),
$$

where $f_{\min }$ is the minimum centre frequency considered, $\Delta f$ is the frequency step used to explore the different frequency values, and $K$ is the number of frequency bands analysed. The maximum centre frequency considered is given by

$$
f_{\text {max }}=f_{\text {min }}+(K-1) \Delta f \text {. }
$$

Equations (4) and (5) define a two-stage procedure for computing the energy of the SOI. Energy (5) is not robust and if $y\left(n T_{s}, f_{c}\right)$ is contaminated by interference, then an anomalous total energy will be estimated.

In order, to obtain robust energy estimation, (5) is generalized and the two-stage detection scheme detailed in Figure 2 is suggested. $K$ realizations of $E\left(f_{c}\right)$ are selected on adjacent frequency bands using the analysis window in Figure 2 and are used to compute a robust decision variable, $D$. $D$ is obtained as the output of a nonlinear combining function:

$$
D=f_{K}\left[E\left(f_{\min }\right), \ldots, E\left(f_{c}\right), \ldots, E\left(f_{\max }\right)\right] .
$$

The first stage of the detection scheme significantly reduces the computational complexity whereas the nonlinear combining strategy adopted for the second stage improves the robustness of the system.

In this paper, we consider the class of $M$-estimators [8] for the design of $f_{K}$ in (7). $M$-estimators have been proposed as robust solutions for estimating the location (mean) of a sequence of random variables [8]. In this respect, $D$ can be interpreted as an estimate of the energy measured on the frequency band

$$
B_{\text {tot }}=K \Delta B \text {, }
$$

where $\Delta B$ is the frequency band of the samples collected for a fixed centre frequency, $f_{c} . \Delta B$ is defined by the capabilities of the front-end used for SS. The standard energy detector in (5) can, for example, be obtained by choosing

$$
f_{K}[\cdot]=\operatorname{mean}(\cdot) \text {. }
$$

Equation (9) will be used as baseline for assessing the robustness of the algorithm selected. In this paper, the sample median [16] is used as a robust location estimator:

$$
\begin{aligned}
& f_{K}[\cdot] \\
& \quad=\operatorname{median}\left[E\left(f_{\min }\right), E\left(f_{\min }+\Delta f\right), \ldots, E\left(f_{\max }\right)\right] .
\end{aligned}
$$

When the number of samples used to compute partial energy (4) is large, then it is possible to invoke the central limit theorem and approximate $E\left(f_{c}\right)$ as a real Gaussian random variable:

$$
\begin{aligned}
& E\left(f_{c}\right) \sim \mathcal{N}\left(2\left(\sigma^{2}+\sigma_{s}^{2}\right)\right. \\
& \left.+A^{2}, \frac{4}{N}\left(\sigma^{2}+\sigma_{s}^{2}\right)\left(A^{2}+\sigma^{2}+\sigma_{s}^{2}\right)\right),
\end{aligned}
$$

where the dependence on $f_{c}$ has been dropped for ease of notation. The mean and variance of $E\left(f_{c}\right)$ are computed in the Appendix.

The presence of an interfering term in (3) increases the mean and variance of the energy measured in a specific channel. From the results presented in Section 7, it is shown that interference is present only at specific frequencies and thus it is reasonable to assume that $A$ is nonzero only for a limited number of bands. For this reason, it is possible to model $A$ as a scaled Bernoulli random variable:

$$
A= \begin{cases}A_{0} & \text { with probability } p \\ 0 & \text { with probability } 1-p .\end{cases}
$$

Equations (11) and (12) define a contaminated model where $p$ is the contamination percentage. The sample median is able to effectively cope with contamination models and thus it has been selected for the robust estimation of the signal energy.

\section{Statistical Characterization}

The performance of a detector is usually characterized in terms of probability of false alarm and probability of detection [12]. The probability of false alarm quantifies the occurrence of type 1 errors, that is, the incorrect detection of signals in an unoccupied frequency band. The probability of detection is the probability of correctly detecting the signal presence. The probability of false alarm is particularly important since it is usually adopted for setting the decision threshold, $T_{h}$. In this section, the impact of interference on the false alarm probability is analysed. In particular, the false alarm probability is defined as

$$
P_{\mathrm{fa}}\left(T_{h}\right)=P\left(D>T_{h} \mid H_{0}\right),
$$

where $H_{0}$ is the null hypothesis defined in (1). The decision threshold is obtained by fixing a target probability of false alarm and inverting (13). 
When standard energy detector (9) is used, and in the absence of interference, the false alarm probability associated to the decision variable, $D_{e}$, is given by

$$
P_{\mathrm{fa}}\left(T_{h}\right)=1-Q\left(\frac{T_{h}-2 \sigma^{2}}{4 \sigma^{4} /(K \cdot N)}\right),
$$

where $Q(\cdot)$ is the Cumulative Density Function (CDF) of a standard Normal Gaussian random variable. Equation (14) has been obtained by considering the fact that, in the absence of interference and under $H_{0}$, the mean and variance of $E\left(f_{c}\right)$ become $2 \sigma^{2}$ and $(4 / N) \sigma^{4}$, respectively. Mean operator (9) leaves the mean of $D_{e}$ unaltered and reduces the variance of $E\left(f_{c}\right)$ by a factor $K$.

The threshold, $T_{h}$, is obtained by fixing $P_{\mathrm{fa}}$ and inverting (14). In the presence of outliers, that is, in the presence of energy spikes due to interference, the actual probability of false alarm significantly increases with respect to the desired value. This performance degradation is analysed here as a function of the contamination percentage, $p$, and the Interference-to-Noise power ratio $(J / N)$ is defined as

$$
\frac{J}{N}=\frac{A^{2}}{2 \sigma^{2}} \text {. }
$$

Although the sample median does not, in general, follow a Gaussian distribution, it is possible to show [16] that a Gaussian approximation can be effectively adopted for large values of $K$. In this case, the variance of $D$ is increased by a factor $\pi / 2$ [16] with respect to the mean operator. This is the loss of efficiency to be paid to improve the robustness of the system. Thus, the Gaussian approximation of the probability of false alarm for the median case is given by

$$
P_{\mathrm{fa}}\left(T_{h}\right)=1-Q\left(\frac{T_{h}-2 \sigma^{2}}{2 \pi \sigma^{4} /(K \cdot N)}\right) .
$$

This probability of false alarm is used in the following paragraph to set the decision threshold in the median case.

The false alarm probability degradation, considering mean and median operators, is shown in Figure 3 where the estimated $P_{\mathrm{fa}}$ is plotted as a function of the desired probabilities, (14) and (16), for a fixed $J / N=0 \mathrm{~dB}$. For the simulations, the parameters reported in Table 1 have been used. These parameters correspond to the values that could be encountered for a typical Digital Video Broadcast-Terrestrial (DVB-T) signal with a total frequency occupation equal to $8 \mathrm{MHz}$. In the absence of degradation, the curves shown in Figure 3 should coincide with the diagonal of the square with corners $(0,0)$ and $(1,1)$ : the higher the distance from such diagonal is, the more the detector is affected by the interfering term. As expected, all the curves in Figure 3 are above the diagonal and thus all the detectors suffer some degradations. The detector based on the median is however significantly less affected by the presence of outliers and only relatively small displacements from the diagonal are observed. Degradations become more and more severe as the contamination factor, $p$, is increased. For $p=0.3$, the mean detector provides an actual false alarm probability practically saturated to 1 : this implies that the detector will always declare the signal present
TABLE 1: Parameters adopted for the simulation performed in Figures 3 and 4 .

\begin{tabular}{lc}
\hline Parameter & Value \\
\hline$K$ & 8 \\
\hline$\Delta B$ & $1 \mathrm{MHz}$ \\
\hline SOI total bandwidth & $8 \mathrm{MHz}$ \\
\hline
\end{tabular}

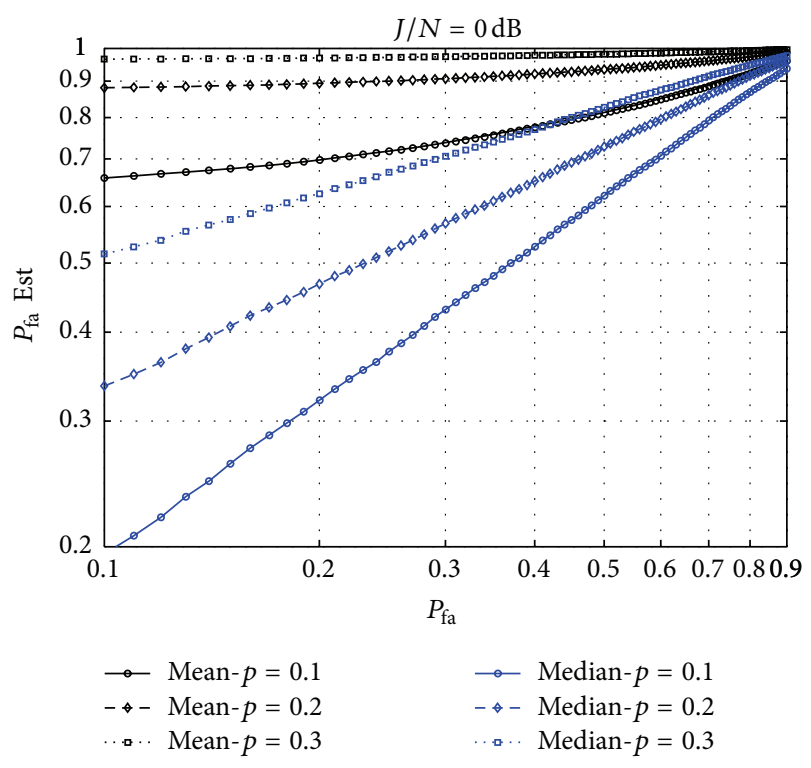

FIgURE 3: Degradation in terms of false alarm probability as a function of the contamination probability, $p$, for a fixed $J / N=0 \mathrm{~dB}$. $\Delta B=1 \mathrm{MHz}, K=8$. Probabilities of false alarm have been plotted in logarithmic scale.

even if the frequency channel is unoccupied. This makes the standard energy detector unusable.

The degradation is analysed for a fixed contamination probability $(p=0.1)$ and for different $J / N$ values, that is -10 , -5 , and $0 \mathrm{~dB}$ in Figure 4. These low $J / N$ values demonstrate the lack of robustness of standard techniques which suffer a significant increase in terms of false alarm probability even in the presence of weak interference. The performance of the median operator does not depend on the $J / N$ of the interfering signal and thus only the worst case is considered in Figure 4.

Curves relative to the standard approach tend to the saturation curve $P_{\text {fa,sat }}$ which can be expressed as

$$
P_{\text {fa,sat }}\left(T_{h}\right)=(1-p)^{K} P_{\mathrm{fa}}+\left(1-(1-p)^{K}\right),
$$

where $P_{\mathrm{fa}}$ is the target probability of false alarm used to set the detection threshold. Equation (17) is obtained by assuming that in the presence of at least one outlier the probability of false alarm is equal to 1 . As expected, the mean operator shows a strong sensitivity to the $J / N$ variations whereas the median is robust to these fluctuations. In particular, from Figure 4 it emerges that the probability of false alarm of the mean energy detector already saturates for $J / N=-5 \mathrm{~dB}$.

The analyses performed in Figures 3 and 4 highlight the fact that the mean shows the greatest performance 


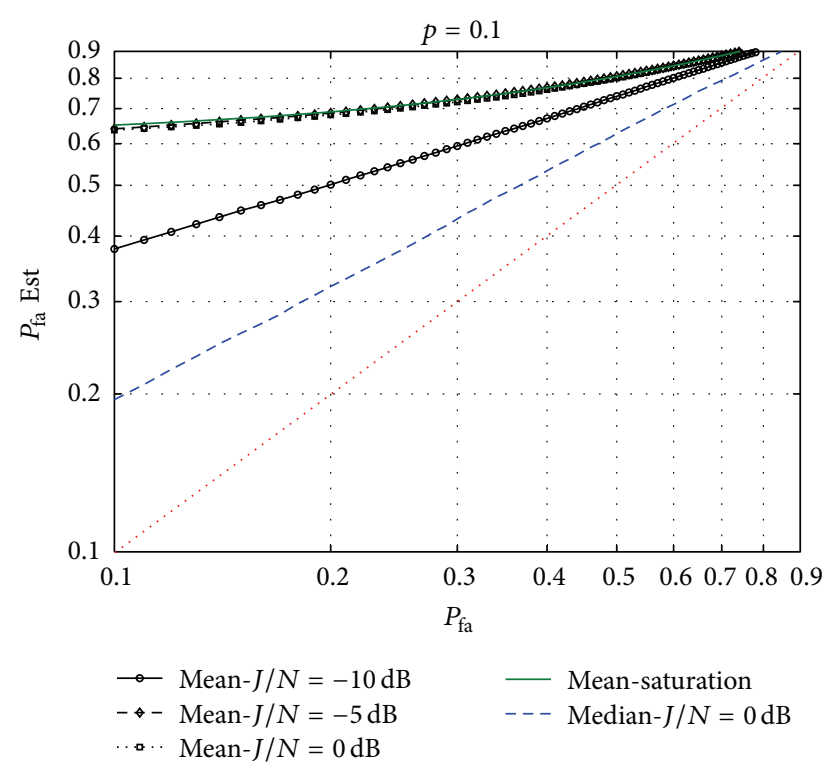

FIgURE 4: Degradation in terms of false alarm probability as a function of the $J / N$ for a fixed contamination probability, $p=0.1$. $\Delta B=1 \mathrm{MHz}, K=8$. Probabilities of false alarm have been plotted in logarithmic scale.

degradation in the presence of interference. These results clearly show the advantages of a robust detector in reducing and controlling the false alarm probability of the system.

\section{System Architecture and Implementation}

In order to prove the benefits of robust energy sensing and the possibility of implementing CR capabilities using a lowcost front-end, a SDR system has been developed using the Realtek RTL2832U device.

The system developed had to meet two basic requirements: (1) being able to operate in real-time and (2) allowing data recording. Real-time operations are necessary to obtain a preliminary analysis of the data streamed by the Realtek RTL2832U device and demonstrate SS whereas data recording is fundamental to allows postmission operations. The samples collected using the Realtek RTL2832U device are valuable data which can be used to design and test new SS algorithms. For this reason, the general architecture depicted in Figure 5 has been adopted. The TV tuner is interfaced to the main software through dedicated drives. The API provided by the OsmocomSDR Project [7] was used in a PC implementation whereas the driver developed by Marinov [17] was adopted for the Android port of the spectrum sensor. The API allows a user to set the different parameters of the TV tuner including the centre and sampling frequencies. A software controller is used to progressively change the signal centre frequency and to start the streaming of the I/Q samples which are stored in a circular buffer. The buffer is accessible via two different threads: the first is used for realtime processing whereas the second is responsible for storing data to disk. (In the Android implementation, data are stored on a Secure Digital (SD) card.) Data are stored according to the format described in Figure 5(a): a synchronization pattern is inserted at the beginning of each data block and it is followed by the centre frequency of the samples. The synchronization pattern is a sequence of 20 bytes whereas the centre frequency is saved as a 32-bit unsigned integer. The I/Q samples are stored in an interleaved way and the size of each data block is a parameter selectable by the user.

This architecture is very generic and allows both real-time and postprocessing operations. The samples provided by the Realtek RTL2832U device are a function of both time and centre frequency and correspond to the samples, $y\left(n T_{s}, f_{c}\right)$, considered in Section 3.

The architecture detailed above has been implemented on both PC and Android platforms. For the PC implementation, SS capabilities have been implemented in the JRC Interference Monitor (JIM) software, a real-time platform is designed for interference monitoring and extended here to process I/Q samples from the Realtek RTL2832U device. JIM has been developed in $\mathrm{C}++$ using the Object Oriented Programming (OOP) paradigm. The main elements of JIM are as follows.

(i) A front-end: a hardware device providing samples to the software. Although this is not an element of the software, JIM has to be aware of the front-end used in order to adopt the correct protocol for data streaming.

(ii) SampleSources: these objects provide a standard software interface to the hardware device connected to JIM. All the sample sources are derived from a base class ("BaseSampleSource") and thus have the same basic interfaces. The main goal of a sample source is to provide the samples to be processed.

(iii) SampleConsumerDataProducers: these objects consume the samples provided by a SampleSource and generate data which can be then displayed. An example is the Histogram Consumer which uses the samples provided by a SampleSource to compute their relative frequencies (probabilities) of the input samples. The Histogram Consumer produces a list of pairs containing the sample value and its relative frequency. These data are then ready to be displayed in some form.

(iv) DataDisplayer: these objects get the data from the SampleConsumerDataProducers and either display them on the screen or save them to a file.

The processing chain describing the principles of operations of JIM is shown in Figure 6. A screen-shot of the JIM software for SS is shown in Figure 7(a). The different views of the software are progressively updated showing the signal evolution as a function of the selected centre frequency.

The Android software has been developed in Java using an architecture similar to that adopted for JIM. The main activity of the Android implementation of the spectrum sensor based on the Realtek RTL2832U front-end is shown in Figure 7(b). The interface allows a user to set different parameters such as the frequency range to sweep and the sampling frequency. The application implements the robust energy detection algorithms detailed in Section 4. 


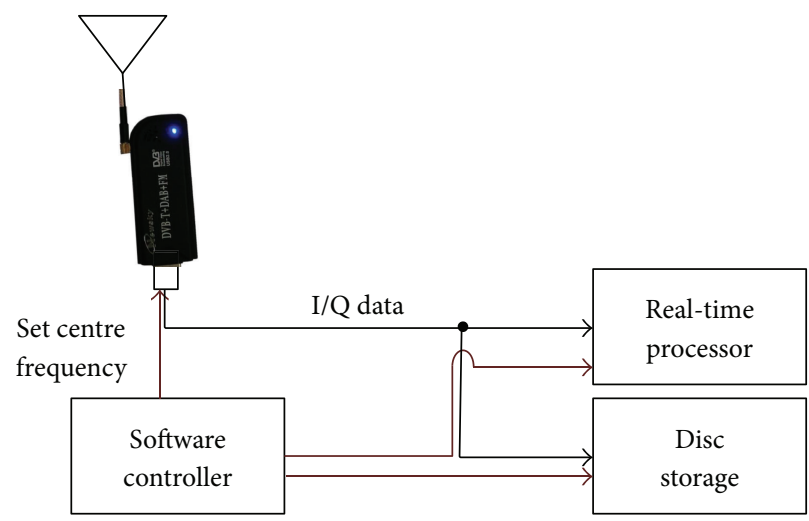

(a)

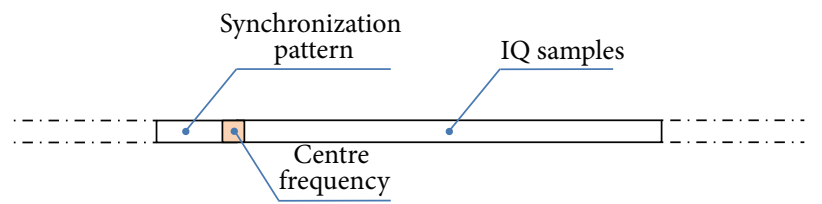

(b)

FIgURE 5: Architecture adopted for the real-time processing and storage of the I/Q samples streamed by the Realtek RTL2832U device. A software controller is used to scan a large frequency band. The data are stored according to the format detailed in the bottom part of the figure.

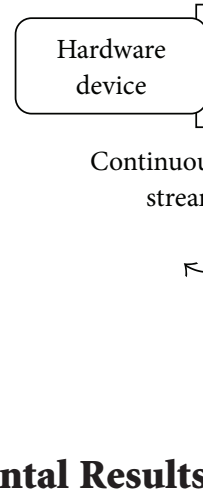

\section{Experimental Results}

Several experiments were conducted to test the capabilities of the system developed and the sample results are provided in this section.

A first set of experiments was conducted in order to experimentally verify the validity of model (3). In particular, we were interested in showing the presence of selfinterference, that is, that form of interference generated by the front-end itself. For this reason, the antenna port of the Realtek device was terminated by a $50 \mathrm{Ohm}$ resistance. Moreover, the device was closed in a box covered by RF absorbers as shown in Figure 8. In this way, it was possible to measure the energy of the signal components generated by the device. In Figure 9, the energy measured in the Ultra-High Frequency (UHF) band is shown when the experimental setup shown in Figure 8 is adopted. The blue curve denoted as "Unfiltered" shows the energy, $E\left(f_{c}\right)$, computed using (4) and with no further processing. The sampling frequency of the Realtek RTL2832U was set to $1.024 \mathrm{MHz}$ in order to obtain a signal bandwidth, $\Delta B$, close to that in Table 1. Spurs and oscillator artefacts are clearly visible; moreover, small variations in the noise floor can be observed as a function of $f_{c}$. These results support the signal model adopted in Section 3. It is possible to observe that spurs occur at regular intervals spaced by 28.8 MHz. This is the fundamental frequency of the crystal oscillator mounted on the Realtek RTL2832U device.

Estimates of the total energy in a $8 \mathrm{MHz}$ bandwidth are also provided in Figure 9. When the mean operator is used for computing the total energy, spurs are still present. These artefacts can bias the detection process. The median operator is an effective tool to remove those artefacts and frequency spikes are no longer present. After spike removal, the energy measured in Figure 9 can be used to estimate the noise 


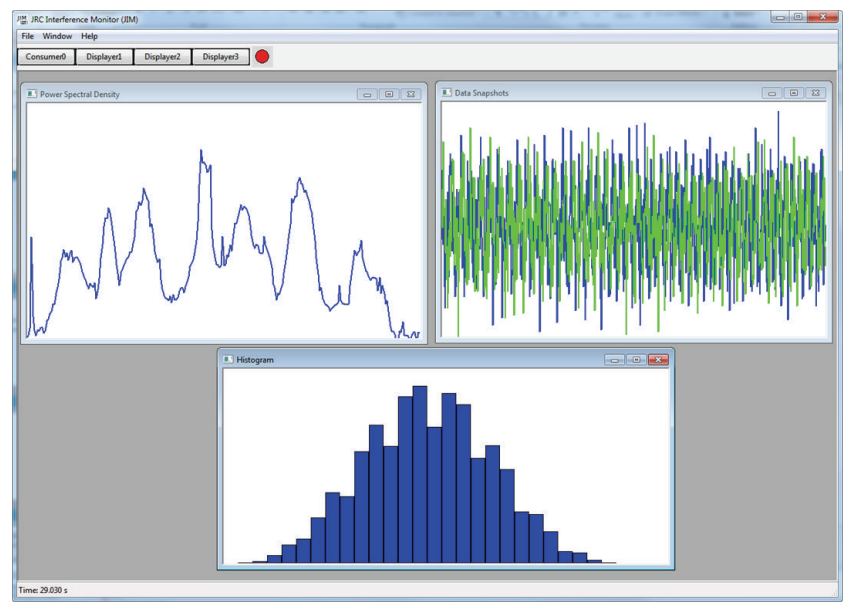

(a)

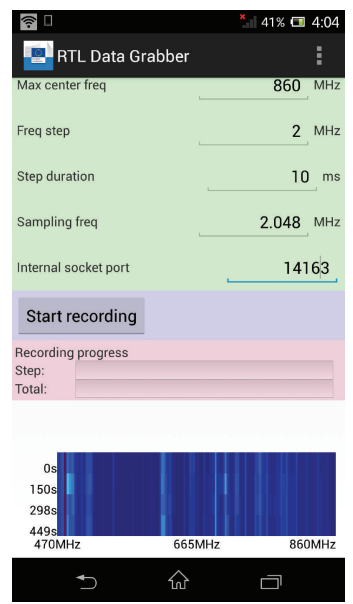

(b)

FIGURE 7: (a) Screen-shot of the JIM software for SS. The different views of the software GUI are progressively updated showing the signal evolution as a function of the selected centre frequency. (b) Main activity of the Android implementation of the spectrum sensor based on the Realtek RTL2832U front-end. The spectrogram showing the energy estimated in the different bands is progressively depicted in the lower part of the GUI.
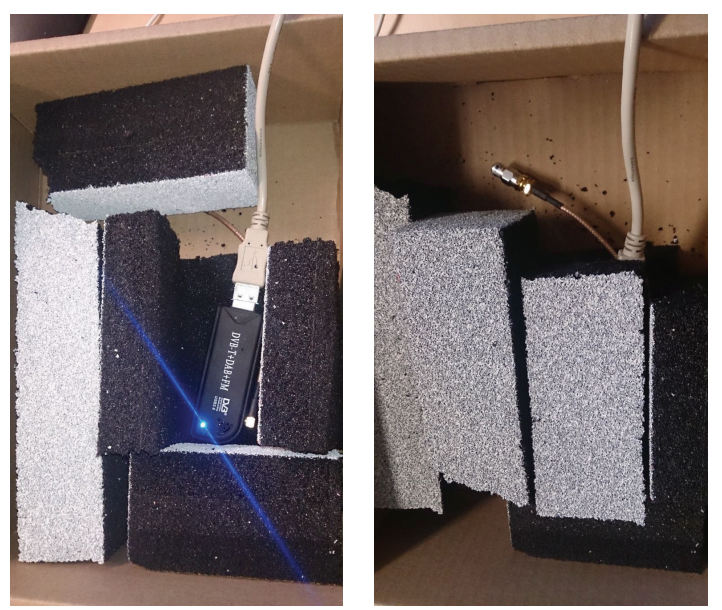

FIGURE 8: The Realtek RTL2832U device was closed inside a box covered by RF absorbers and with the antenna port closed by a 50 Ohm terminator.

variance, $\sigma^{2}$, as a function of the centre frequency, $f_{c}$. In particular, $\sigma^{2}$ has been estimated using the samples collected using this setup, that is, in the absence of signal, and accounting for the presence of interference. When the signal is absent, $\sigma^{2}$ can be estimated using (7) where the median has been adopted to remove the impact of interference.

Note that the suitability of the Realtek RTL2832U device for energy detection depends on the frequency band considered. For example, when the L band ([1100-1700] MHz) is considered, the Realtek RTL2832U front-end has a significantly worse behaviour than in the UHF band. This fact is highlighted in Figure 10 where the noise energy measured by the Realtek RTL2832U device is shown as a function of the centre frequency. Also in this case, the antenna port of

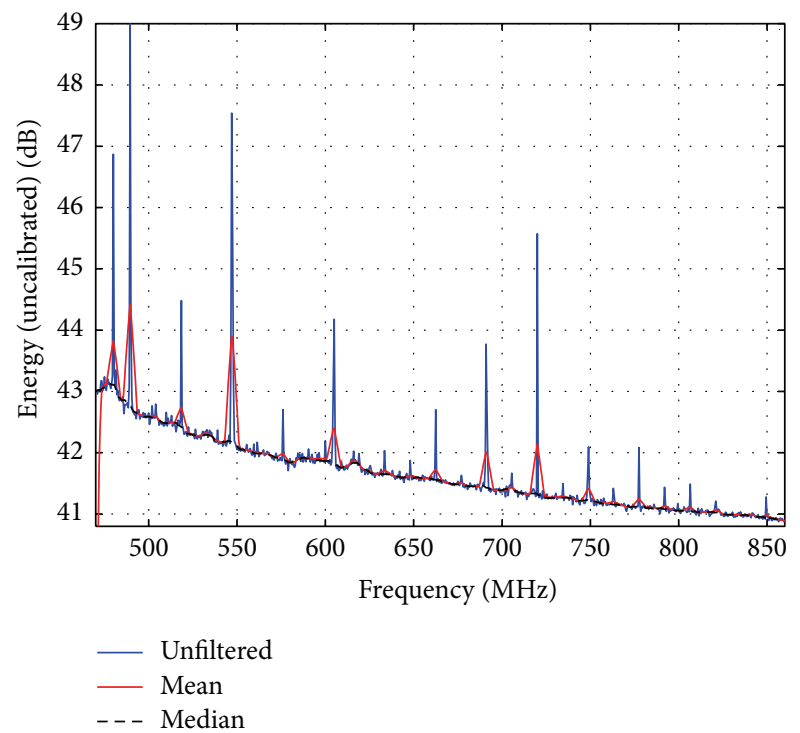

FIGURE 9: Energy measured in the UHF band when the antenna port of the Realtek RTL2832U device is terminated using a $50 \mathrm{Ohm}$ resistance. $\Delta B=1 \mathrm{MHz}, K=8$.

the device was terminated with a $50 \mathrm{Ohm}$ resistance and the TV tuner was covered by RF signal absorbers. Several spikes are present and the noise floor does not show a regular trend. In this case, the frequency spikes are approximately 14.4 MHz, half the frequency measured for the UHF bands. The median operator is able to effectively remove most of the spikes present in the energy profile in Figure 10.

The detection capabilities of the device have been tested using real and simulated data. Simulations were performed using a hardware-in-the-loop approach. In particular, a Universal Software Radio Platform (USRP) 2 device was used 


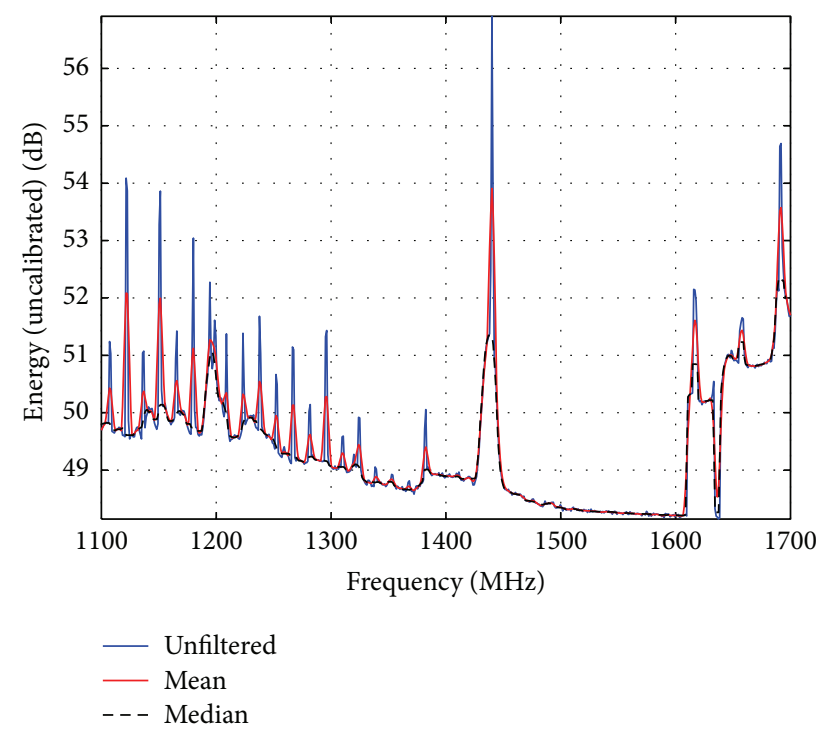

FIGURE 10: Energy measured in the L band when the antenna port of the Realtek RTL2832U device is terminated using a $50 \mathrm{Ohm}$ resistance. $\Delta B=1 \mathrm{MHz}, K=8$.

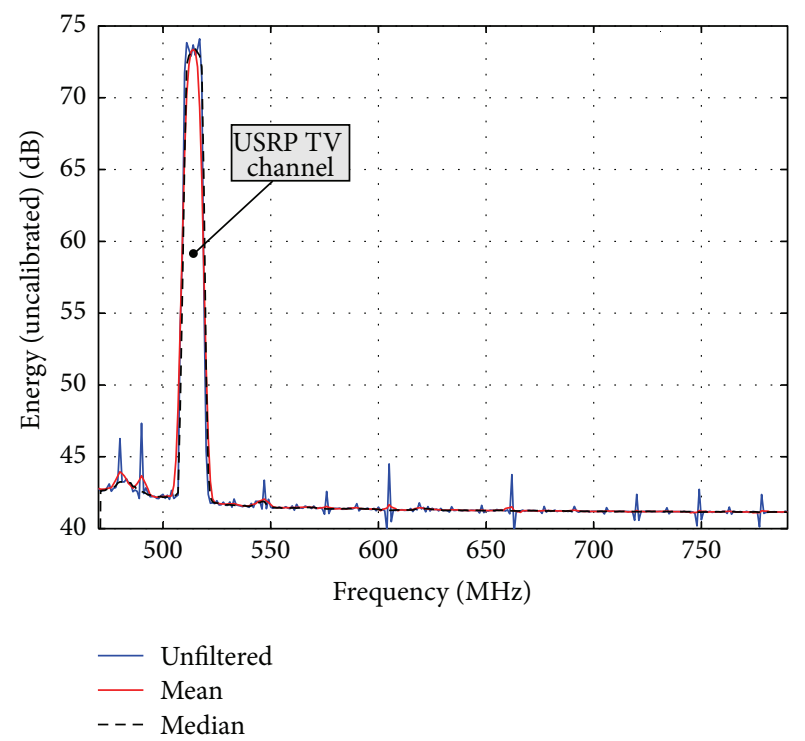

FIGURE 11: Energy measured in the UHF band when the antenna port of the Realtek RTL282U device is connected to a USRP simulating a single TV channel. $\Delta B=1 \mathrm{MHz}, K=8$.

to generate a useful signal component with the same spectral characteristics of a TV channel. An example of the curves obtained with this approach is shown in Figure 11. Here, the signal presence is clearly detected. The channels adjacent to the useful signal are only marginally affected by its presence and the noise floor is consistent with the values measured in Figure 9.

\section{Conclusions}

In this paper, SS has been demonstrated using a low cost TV tuner. In particular, a Realtek RTL2832U device was used as an agile front-end for scanning the UHF bands. A twostage energy detection scheme was proposed to improve the robustness of the system and to deal with the spurs and imperfections caused by the front-end oscillator. The use of robust estimators, such as the median filter, allows a significant improvement, in terms of false alarm probability, with respect to standard techniques. Real data and hardware-inloop simulations were used to test the system developed and to characterize the performance of the algorithm proposed. The advantages of robust detection techniques were clearly shown.

The use of robust estimators can also be used in the presence of narrowband wireless interference like CWs. The application of the algorithms used in this paper in the presence of wireless interference will be addressed in future work. Future work also includes a better characterization of the front-end with specific focus on the impact of Automatic Gain Control (AGC) and the study of additional robust techniques such as the myriad filter [8].

\section{Appendix}

\section{Mean and Variance of $E\left(f_{c}\right)$}

In this Appendix, the mean and variance of $E\left(f_{c}\right)$ are evaluated. For the analysis $i\left(n T_{s}, f_{c}\right)$ is assumed to be a complex CW:

$$
i\left(n T_{s}, f_{c}\right)=\exp \left\{j 2 \pi f_{i} n T_{s}+j \varphi_{i}\right\},
$$

where $f_{i}$ is the interference centre frequency and $\varphi_{i}$ is a uniform random variable which spans the interval $[-\pi, \pi]$. The properties derived in this section are the basis of (11).

The mean of $E\left(f_{c}\right)$ can be computed as

$$
\begin{aligned}
& \mathrm{E}\left[\mathrm{E}\left(f_{c}\right)\right]=\mathrm{E}\left[\frac{1}{N} \sum_{n=0}^{N-1}\left|y\left(n T_{s}, f_{c}\right)\right|^{2}\right]=\frac{1}{N} \\
& \quad \cdot \sum_{n=0}^{N-1}\left\{\sigma_{s}^{2} \mathrm{E}\left[\left|s\left(n T_{s}, f_{c}\right)\right|^{2}\right]\right. \\
& +A^{2}\left(f_{c}\right) \mathrm{E}\left[\left|i\left(n T_{s}, f_{c}\right)\right|^{2}\right]+\mathrm{E}\left[\left|\eta\left(n T_{s}, f_{c}\right)\right|^{2}\right] \\
& +2 \sigma_{s} A\left(f_{c}\right) \mathrm{E}\left[\Re\left\{s\left(n T_{s}, f_{c}\right) i^{*}\left(n T_{s}, f_{c}\right)\right\}\right] \\
& +2 \sigma_{s} \mathrm{E}\left[\Re\left\{s\left(n T_{s}, f_{c}\right) \eta^{*}\left(n T_{s}, f_{c}\right)\right\}\right] \\
& \left.+2 A\left(f_{c}\right) \mathrm{E}\left[\Re\left\{\eta\left(n T_{s}, f_{c}\right) i^{*}\left(n T_{s}, f_{c}\right)\right\}\right]\right\} .
\end{aligned}
$$

The three components in (3) are independent and zero mean. Thus the three cross-terms in (A.2) are zero mean and $\mathrm{E}\left[E\left(f_{c}\right)\right]$ becomes

$$
\mathrm{E}\left[E\left(f_{c}\right)\right]=2 \sigma_{s}^{2}+2 \sigma^{2}+A^{2}\left(f_{c}\right) .
$$


The variance of $E\left(f_{c}\right)$ is given by

$$
\begin{aligned}
& \operatorname{Var}\left[E\left(f_{c}\right)\right]=\operatorname{Var}\left[\frac{1}{N} \sum_{n=0}^{N-1}\left|y\left(n T_{s}, f_{c}\right)\right|^{2}\right]=\frac{1}{N^{2}} \\
& \quad \cdot \sum_{n=0}^{N-1} \operatorname{Var}\left\{\sigma_{s}^{2}\left|s\left(n T_{s}, f_{c}\right)\right|^{2}+A^{2}\left(f_{c}\right)\left|i\left(n T_{s}, f_{c}\right)\right|^{2}\right. \\
& +\left|\eta\left(n T_{s}, f_{c}\right)\right|^{2} \\
& +2 \sigma_{s} A\left(f_{c}\right) \Re\left\{s\left(n T_{s}, f_{c}\right) i^{*}\left(n T_{s}, f_{c}\right)\right\} \\
& +2 \sigma_{s} \Re\left\{s\left(n T_{s}, f_{c}\right) \eta^{*}\left(n T_{s}, f_{c}\right)\right\} \\
& \left.+2 A\left(f_{c}\right) \Re\left\{\eta\left(n T_{s}, f_{c}\right) i^{*}\left(n T_{s}, f_{c}\right)\right\}\right\} .
\end{aligned}
$$

In (A.4), the variance operator has been moved after the sum used for computing the energy. This operation is possible since $s\left(n T_{s}, f_{c}\right)$ and $\eta\left(n T_{s}, f_{c}\right)$ are assumed to be white sequences. Although $i\left(n T_{s}, f_{c}\right)$ is a highly correlated sequence, it appears in the variance computation multiplied by $s\left(n T_{s}, f_{c}\right)$ and by $\eta\left(n T_{s}, f_{c}\right)$. In this case, the resulting sequences are white. Moreover, term $\left|i\left(n T_{s}, f_{c}\right)\right|^{2}$ is equal to 1 and thus has no impact on the variance computation. These properties justify the commutation between the summation and the variance operator. In addition to this, the six terms in (A.4) are uncorrelated and thus the total variance is equal to the sum of the variances of such components. In particular,

$$
\begin{aligned}
\operatorname{Var} & \left\{\sigma_{s}^{2}\left|s\left(n T_{s}, f_{c}\right)\right|^{2}\right\} \\
& =\sigma_{s}^{4} \operatorname{Var}\left\{s_{i}^{2}\left(n T_{s}, f_{c}\right)+s_{q}^{2}\left(n T_{s}, f_{c}\right)\right\} \\
& =2 \operatorname{Var}\left\{s_{i}^{2}\left(n T_{s}, f_{c}\right)\right\} \\
& =2 \sigma_{s}^{4}\left\{\mathrm{E}\left[s_{i}^{4}\left(n T_{s}, f_{c}\right)\right]-\mathrm{E}^{2}\left[s_{i}^{2}\left(n T_{s}, f_{c}\right)\right]\right\} \\
& =2 \sigma_{s}^{4}[3-1]=4 \sigma_{s}^{4},
\end{aligned}
$$

where $s_{i}\left(n T_{s}, f_{c}\right)$ and $s_{q}\left(n T_{s}, f_{c}\right)$ are the real and imaginary parts of the useful signal, $s\left(n T_{s}, f_{c}\right)$. Similarly, it is possible to prove

$$
\operatorname{Var}\left\{\left|\eta\left(n T_{s}, f_{c}\right)\right|^{2}\right\}=4 \sigma^{4}
$$

The interference term has a constant modulus and thus its variance is zero. The variance of the cross-terms involving $i\left(n T_{s}, f_{c}\right)$ can be computed as

$$
\begin{aligned}
\operatorname{Var} & \left\{2 \sigma_{s} A\left(f_{c}\right) \Re\left\{s\left(n T_{s}, f_{c}\right) i^{*}\left(n T_{s}, f_{c}\right)\right\}\right\} \\
& =4 \sigma_{s}^{2} A^{2}\left(f_{c}\right) \cdot \frac{1}{2} \operatorname{Var}\left\{s\left(n T_{s}, f_{c}\right) i^{*}\left(n T_{s}, f_{c}\right)\right\} \\
& =4 \sigma_{s}^{2} A^{2}\left(f_{c}\right) .
\end{aligned}
$$

The rotation on $s\left(n T_{s}, f_{c}\right)$ performed through the multiplication by $i^{*}\left(n T_{s}, f_{c}\right)$ has no impact on the variance because of the assumption of circular symmetry. Similarly,

$$
\begin{aligned}
\operatorname{Var} & \left\{2 A\left(f_{c}\right) \Re\left\{\eta\left(n T_{s}, f_{c}\right) i^{*}\left(n T_{s}, f_{c}\right)\right\}\right\} \\
& =4 \sigma^{2} A^{2}\left(f_{c}\right) .
\end{aligned}
$$

Finally, the signal and noise cross-term has variance

$$
\operatorname{Var}\left\{2 \sigma_{s} \mathfrak{R}\left\{s\left(n T_{s}, f_{c}\right) \eta^{*}\left(n T_{s}, f_{c}\right)\right\}\right\}=8 \sigma_{s}^{2} \sigma^{2} .
$$

Using these results, it is finally possible to compute the variance of $E\left(f_{c}\right)$ :

$$
\begin{aligned}
\operatorname{Var} & {\left[E\left(f_{c}\right)\right] } \\
& =\frac{1}{N}\left(4 \sigma_{s}^{4}+4 \sigma^{4}+4 A^{2}\left(f_{c}\right)\left(\sigma_{s}^{2}+\sigma^{2}\right)+8 \sigma_{s}^{2} \sigma^{2}\right) \\
& =\frac{4}{N}\left(\sigma_{s}^{2}+\sigma^{2}\right)\left(\sigma_{s}^{2}+\sigma^{2}+A\left(f_{c}\right)\right) .
\end{aligned}
$$

\section{Conflict of Interests}

The authors declare that there is no conflict of interests regarding the publication of this paper.

\section{References}

[1] S. Haykin, "Cognitive radio: brain-empowered wireless communications," IEEE Journal on Selected Areas in Communications, vol. 23, no. 2, pp. 201-220, 2005.

[2] T. Yücek and H. Arslan, "A survey of spectrum sensing algorithms for cognitive radio applications," IEEE Communications Surveys and Tutorials, vol. 11, no. 1, pp. 116-130, 2009.

[3] F. Gao, J. Li, T. Jiang, and W. Chen, "Sensing and recognition when primary user has multiple transmit power levels," IEEE Transactions on Signal Processing, vol. 63, no. 10, pp. 2704-2717, 2015.

[4] E. Rebeiz and D. Cabric, "How wideband receiver nonlinearities impact spectrum sensing," in Proceedings of the 1st IEEE Global Conference on Signal and Information Processing (GlobalSIP '13), pp. 1178-1181, Austin, Tex, USA, December 2013.

[5] E. Rebeiz, A. S. H. Ghadam, M. Valkama, and D. Cabric, "Suppressing RF front-end nonlinearities in wideband spectrum sensing," in Proceedings of the 8th International Conference on Cognitive Radio Oriented Wireless Networks and Communications (CROWNCOM '13), pp. 87-92, July 2013.

[6] S.-K. Ting, D. Cabric, and A. Sayed, "An algorithm to compensate the effects of spurious PLL tones in spectrum sensing architectures," IEEE Transactions on Circuits and Systems. I. Regular Papers, vol. 59, no. 5, pp. 1093-1106, 2012.

[7] OsmocomSDR Project, 2015, http://sdr.osmocom.org/trac/wiki/ rtl-sdr.

[8] G. R. Arce, Nonlinear Signal Processing: A Statistical Approach, Wiley-Interscience, Hoboken, NJ, USA, 2004.

[9] S. Dikmese, P. C. Sofotasios, T. Ihalainen, M. Renfors, and M. Valkama, "Efficient energy detection methods for spectrum sensing under non-flat spectral characteristics," IEEE Journal on Selected Areas in Communications, vol. 33, no. 5, pp. 755-770, 2015.

[10] E. Axell, G. Leus, E. G. Larsson, and H. V. Poor, "Spectrum sensing for cognitive radio: state-of-the-art and recent advances," IEEE Signal Processing Magazine, vol. 29, no. 3, pp. 101-116, 2012.

[11] R. Dionísio, J. Ribeiro, P. Marques et al., "Sensing algorithms for TVWS operations," Project Deliverable D4.2, COGEU FP7 ICT-2009.1.1, 2011.

[12] S. M. Kay, Fundamentals of Statistical Signal Processing, Volume 2: Detection Theory, vol. 1, Prentice Hall, Upper Saddle River, NJ, USA, 1998. 
[13] P. W. Ward, "Design technique for precise GNSS receiver postcorrelation noise floor measurements with usage design examples by the search and tracking processes," in Proceedings of the International Technical Meeting of The Institute of Navigation, pp. 607-617, San Diego, Calif, USA, January 2010.

[14] J. Jiang, H. Sun, D. Baglee, and V. Poor, "Achieving autonomous compressive spectrum sensing for cognitive radios," IEEE Transactions on Vehicular Technology, 2015.

[15] S. Wei, D. L. Goeckel, and P. A. Kelly, "Convergence of the complex envelope of bandlimited OFDM signals," IEEE Transactions on Information Theory, vol. 56, no. 10, pp. 4893-4904, 2010.

[16] R. A. Maronna, R. D. Martin, and V. J. Yohai, Robust Statistics: Theory and Methods, John Wiley \& Sons, Hoboken, NJ, USA, 2006.

[17] M. Marinov, "RTL2832U driver-rtl tcp and libusb-1.0 port for Android modified to support opening devices from Linux file descriptors," 2014, https://github.com/martinmarinov/rtl_tcp_ andro-. 

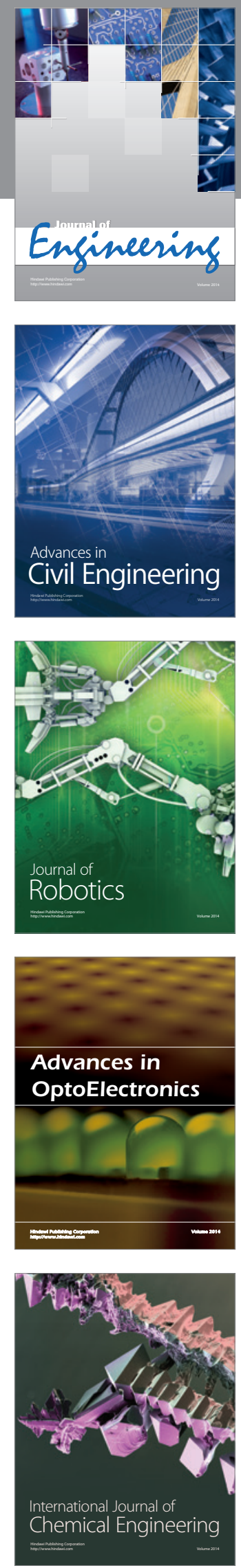

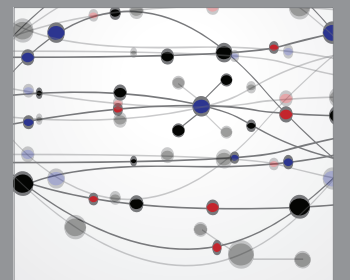

The Scientific World Journal
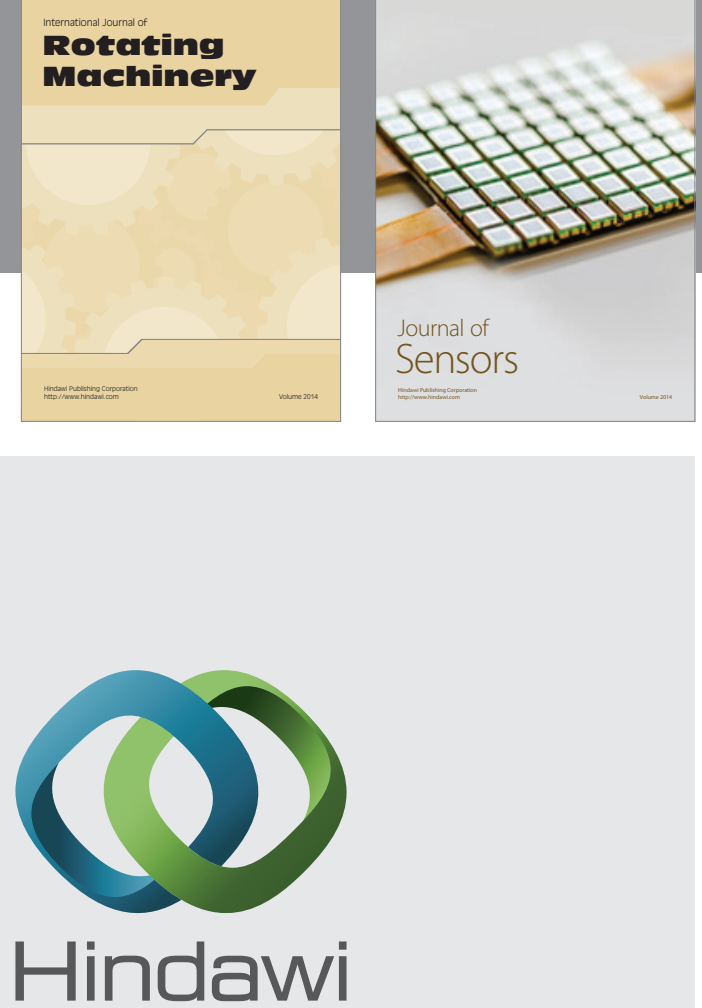

Submit your manuscripts at http://www.hindawi.com
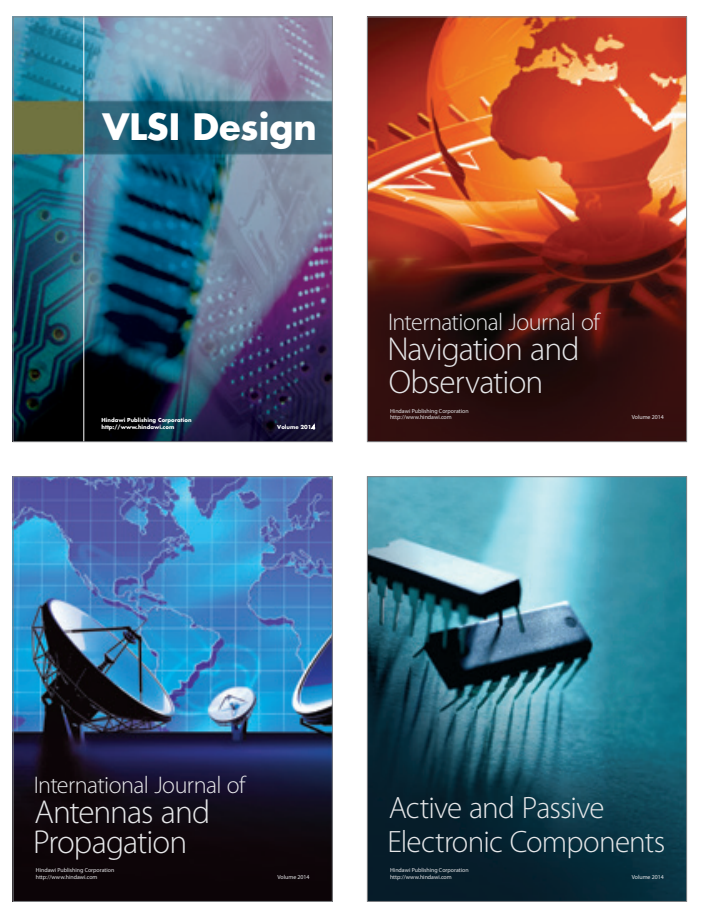
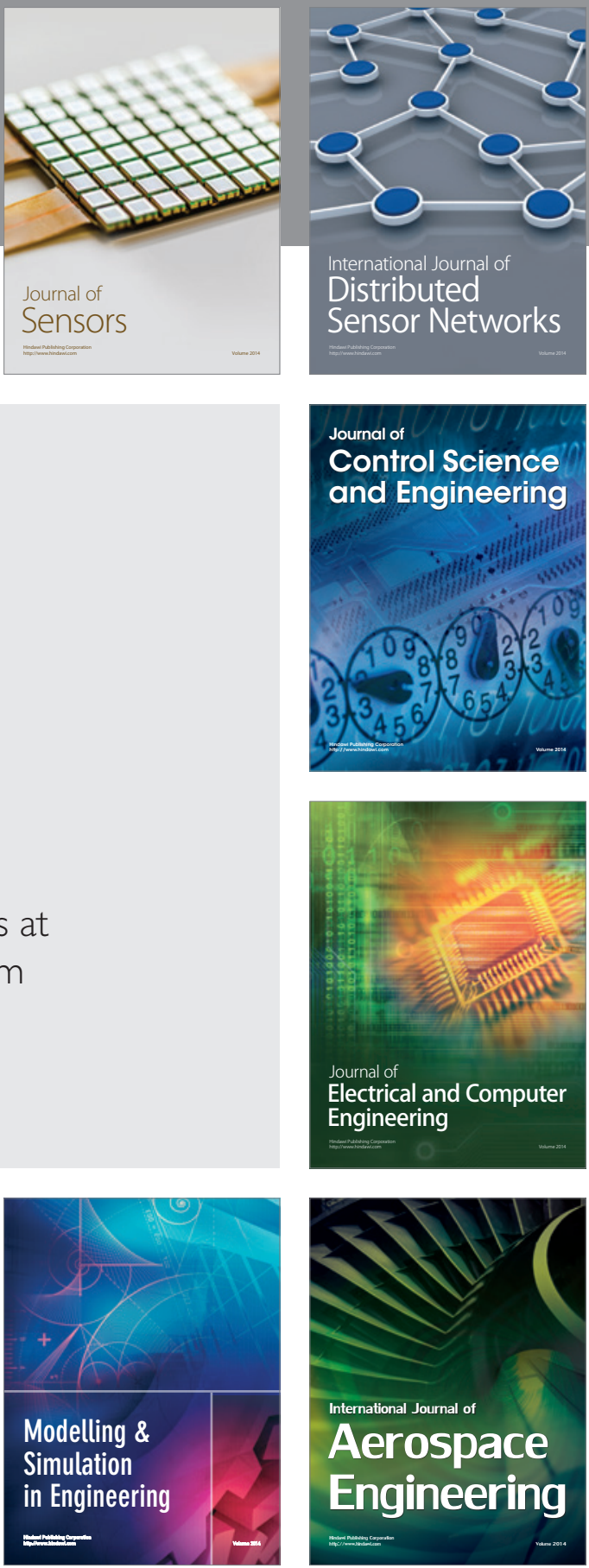

Journal of

Control Science

and Engineering
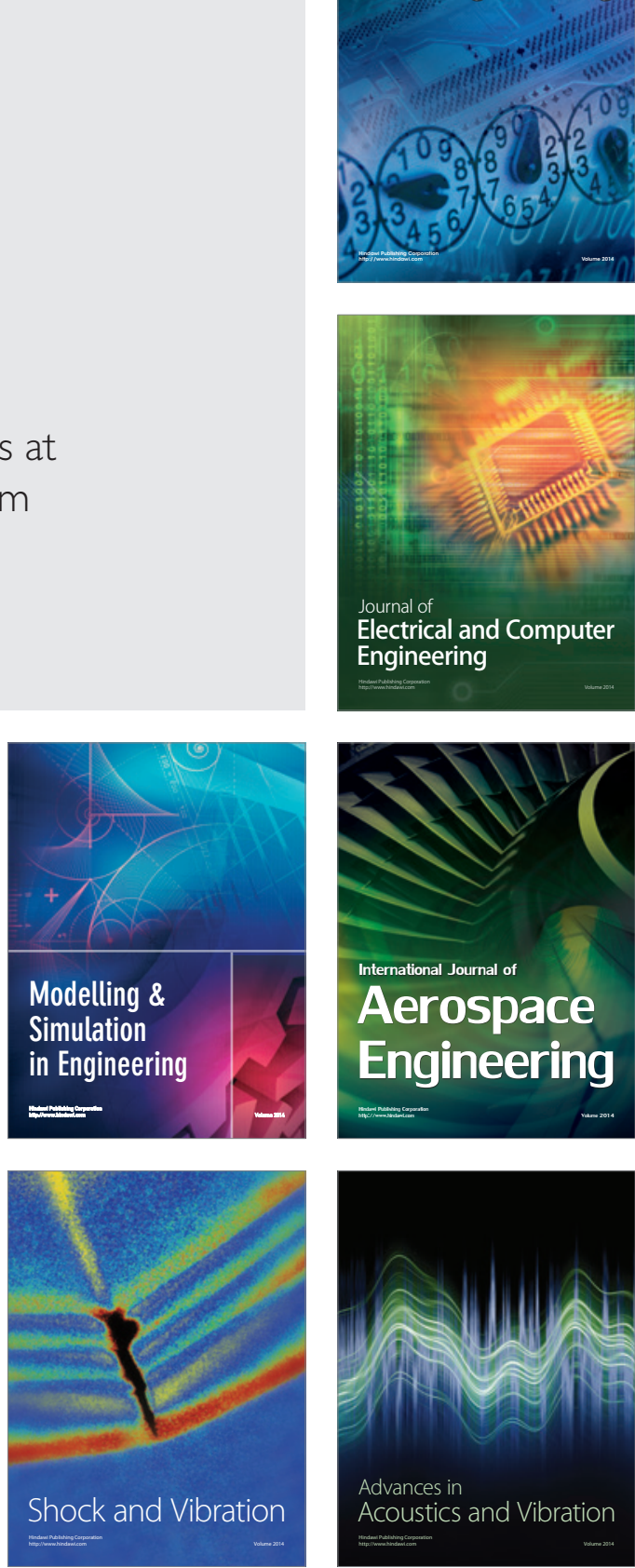